\title{
Human longevity and variation in GH/IGF-1/insulin signaling, DNA damage signaling and repair and pro/antioxidant pathway genes: Cross sectional and longitudinal studies
}

\author{
Mette Soerensen ${ }^{\mathrm{a}, \mathrm{b}}$, Serena Dato ${ }^{\mathrm{a}, \mathrm{c}}$, Qihua Tan ${ }^{\mathrm{a}, \mathrm{b}}$, Mikael Thinggaard a , Rabea Kleindorp ${ }^{\mathrm{d}}$, \\ Marian Beekman ${ }^{\text {eff }}$, Rune Jacobsen ${ }^{\text {a }}$, H. Eka D. Suchiman ${ }^{\text {e,f }}$, Anton J.M. de Craen ${ }^{\mathrm{g}}$, Rudi G.J. Westendorp ${ }^{\mathrm{g}}$, \\ Stefan Schreiber ${ }^{\mathrm{d}}$, Tinna Stevnsner ${ }^{\mathrm{h}}$, Vilhelm A. Bohr ${ }^{\mathrm{h}, \mathrm{i}}$, P. Eline Slagboom ${ }^{\mathrm{e}, \mathrm{f}}$, Almut Nebel ${ }^{\mathrm{d}}$, \\ James W. Vaupel ${ }^{\mathrm{a}, \mathrm{j}}$, Kaare Christensen ${ }^{\mathrm{a}, \mathrm{b}}$, Matt McGue ${ }^{\mathrm{a}, \mathrm{k}}$, Lene Christiansen ${ }^{\mathrm{a}, \mathrm{b}, *}$ \\ ${ }^{a}$ The Danish Aging Research Center, Epidemiology, Institute of Public Health, University of Southern Denmark, J.B. Winsloews Vej 9B, 5000 Odense C, Denmark \\ ${ }^{b}$ Department of Clinical Genetics and Department of Clinical Biochemistry and Pharmacology, Odense University Hospital, Sdr. Boulevard 29, 5000 Odense C, Denmark \\ ' Department of Cell Biology, University of Calabria, Ponte Pietro Bucci cubo 4C, 87036 Rende (Cs), Italy \\ d Institute of Clinical Molecular Biology, Christian-Albrechts-University and University Hospital Schleswig-Holstein, Schittenhelmstrasse 12, 24105 Kiel, Germany \\ e Department of Molecular Epidemiology, Leiden University Medical Center, S5-P, PO Box 9600, 2300 RC Leiden, The Netherlands \\ ${ }^{\mathrm{f}}$ Netherlands Consortium for Healthy Aging, Leiden University Medical Center, 2300 RC, Leiden, The Netherlands \\ ${ }^{g}$ Department of Gerontology and Geriatrics, Leiden University Medical Center, C2-R, PO Box 9600, 2300 RC Leiden, The Netherlands \\ h The Danish Aging Research Center, Department of Molecular Biology and Genetics, Aarhus University, C. F. Moellers Allé 3, 8000 Aarhus C, Denmark \\ i Laboratory of Molecular Gerontology, National Institute on Aging, National Institute of Health, 5600 Nathan Shock Drive, 21224-6825 Baltimore, Maryland, USA \\ j Max Planck Institute for Demographic Research, Konrad-Zuse-Str. 1, 18057 Rostock, Germany \\ k Department of Psychology, University of Minnesota, N218 Elliott Hall 75 East River Road, MN 55455-0344 Minneapolis, MN, USA
}

\section{A R T I C L E I N F O}

\section{Article history:}

Received 25 October 2011

Received in revised form 22 February 2012

Accepted 24 February 2012

Available online 3 March 2012

Section Editor: T.E. Johnson

\section{Keywords:}

Human longevity

Association study

Case-control data

Longitudinal data

Candidate study

\begin{abstract}
A B S T R A C T
Here we explore association with human longevity of common genetic variation in three major candidate pathways: GH/IGF-1/insulin signaling, DNA damage signaling and repair and pro/antioxidants by investigating 1273 tagging SNPs in 148 genes composing these pathways.

In a case-control study of 1089 oldest-old (age 92-93) and 736 middle-aged Danes we found 1 pro/antioxidant SNP (rs1002149 (GSR)), 5 GH/IGF-1/INS SNPs (rs1207362 (KL), rs2267723 (GHRHR), rs3842755 (INS), rs572169 (GHSR), rs9456497 (IGF2R)) and 5 DNA repair SNPs (rs11571461 (RAD52), rs13251813 (WRN), rs1805329 (RAD23B), rs2953983 (POLB), rs3211994 (NTLH1)) to be associated with longevity after correction for multiple testing. In a longitudinal study with 11 years of follow-up on survival in the oldest-old Danes we found 2 pro/antioxidant SNPs (rs10047589 (TNXRD1), rs207444 (XDH)), 1 GH/IGF-1/INS SNP (rs26802 (GHRL)) and 3 DNA repair SNPs (rs13320360 (MLH1), rs2509049 (H2AFX) and rs705649 (XRCC5)) to be associated with mortality in late life after correction for multiple testing.

When examining the 11 SNPs from the case-control study in the longitudinal data, rs3842755 (INS), rs13251813 (WRN) and rs3211994 (NTHL1) demonstrated the same directions of effect $(\mathrm{p}<0.05)$, while rs9456497 (IGF2R) and rs1157146 (RAD52) showed non-significant tendencies, indicative of effects also in late life survival. In addition, rs207444 $(X D H)$ presented the same direction of effect when inspecting the 6 SNPs from the longitudinal study in the case-control data, hence, suggesting an effect also in survival from middle age to old age.

No formal replications were observed when investigating the 11 SNPs from the case-control study in 1613 oldestold (age 95-110) and 1104 middle-aged Germans, although rs11571461 (RAD52) did show a supportive nonsignificant tendency $(\mathrm{OR}=1.162,95 \% \mathrm{Cl}=0.927-1.457)$. The same was true for rs10047589 (TNXRD1) $(\mathrm{HR}=0.758,95 \% \mathrm{CI}=0.543-1.058)$ when examining the $6 \mathrm{SNPs}$ from the longitudinal study in a Dutch longitudinal cohort of oldest-old (age $85+, \mathrm{N}=563$ ).

In conclusion, the present candidate gene based association study, the largest to date applying a pathway approach, not only points to potential new longevity loci, but also underlines the difficulties of replicating association findings in independent study populations and thus the difficulties in identifying universal longevity polymorphisms.
\end{abstract}

(c) 2012 Elsevier Inc. All rights reserved.

\footnotetext{
* Corresponding author at: Epidemiology, Institute of Public Health, University of Southern Denmark, J.B. Winsloews Vej 9B, 5000 Odense C, Denmark. Tel.: +45 65503378; fax: + 4565503682 .

E-mail address: lchristiansen@health.sdu.dk (L. Christiansen).
}

\section{Introduction}

Genetic factors contribute approximately $25 \%$ to variation in human life span (Herskind et al., 1996), an effect thought to be 
minimal before age 60 years and most profound from age 85 years and onwards (Hjelmborg et al., 2006). Candidate longevity genes encode proteins involved in several biological processes including lipidprotein metabolism (APOE being by far the most validated longevity gene to date), growth hormone 1 /insulin-like growth factor 1 /insulin (GH/IGF-1/INS) signaling, DNA damage signaling, and repair and pro/ antioxidant pathways (Christensen et al., 2006). The genes composing the latter three pathways encode proteins involved in processes such as energy metabolism, growth and differentiation, and the maintenance of genetic stability (including telomere stability), and protection against free radicals produced by the general metabolism. These are all processes believed to be of key importance for aging and longevity from nematodes to humans (reviewed in e.g. Burtner and Kennedy, 2010; Cluett and Melzer, 2009; Harman, 1973; Kenyon, 2010; Kuningas et al., 2008). Studies using various animal models have found that either depletion or over-expression of some of these candidate genes affect the life span of the organism (e.g. $G H$, GPX1, FOXO, KL, GHRHR, POLB, RAD52, INS and TP53 (Bartke, 2005; Cabelof et al., 2006; Flurkey et al., 2001; Giannakou et al., 2004; Holzenberger et al., 2003; Kawano et al., 2000; Kuro-o et al., 1997; Kurosu et al., 2005; Park et al., 1999; Tyner et al., 2002; Mockett et al., 1999)), whereas manipulating other genes leads to aging-like phenotypes (e.g. PON1 and CSB (Shih et al., 1998; van der Horst et al., 1997)).

In humans rare mutations that severely affect the function of some of these genes (WRN, CSB and RECQL4) can cause segmental progeroid premature aging syndromes such as Werner's, Cockayne's and Rothmund-Thomson syndromes (reviewed in Burtner and Kennedy, 2010)), whereas more common genetic variation has been reported to be associated with human lifespan. Examples from the GH/IGF-1/INS pathway include IGF1R, GHR and FOXO3A, from the DNA damage signaling and repair pathway WRN, MLH1 and TP53 and from the pro/antioxidant pathway SOD1, SOD2 and PON1 (Bonafe et al., 2003; Castro et al., 2000; Christiansen et al., 2004; Flachsbart et al., 2009a; Kim et al., 2006; van Heemst et al., 2005a; van Heemst et al., 2005b; Willcox et al., 2008a; Yashin et al., 2000).

The vast majority of the candidate genetic association studies published so far focus on a single or a few single nucleotide polymorphisms (SNPs) or candidate genes, although recently a few studies have initiated the investigation of SNPs in groups of candidate genes and pathways based on the functional roles of the gene products (Deelen et al., 2011; Flachsbart et al., 2010; Nebel et al., 2009; Pawlikowska et al., 2009a). In addition, previous studies have generally applied a case-control study design, thus raising concerns of bias introduced by differences in characteristics of cases and controls, for example cohort effects.

Here we investigate the association of common genetic variation in 148 genes composing the GH/IGF-1/INS signaling, DNA damage signaling and DNA repair and pro/antioxidant pathways with human longevity. The study populations are a cohort of Danes born in 1905 and followed from age 93 years until death, as well as a group of middle-aged controls. This provides a unique opportunity to investigate these variations in key candidate genes of human longevity using both case-control and longitudinal designs. Further, the SNPs identified in the case-control study are explored in the longitudinal data in order to investigate whether these SNPs also pose effects on survival during old age, and the SNPs identified in the longitudinal study are examined in the case-control data to investigate whether these SNPs also pose effects on survival from middle age to old age.

Finally, considering the importance of replicating initial results in genetic association studies, the SNPs found to be significant in the case-control study are tested for association in a German casecontrol sample (1613 long-lived individuals and 1104 controls), while the findings from the Danish longitudinal study are tested in the prospective Dutch Leiden 85-plus Study (563 individuals).

\section{Material and methods}

\subsection{Subjects}

\subsubsection{Discovery cohorts}

The study population of oldest-old was 1200 participants from The Danish 1905 Birth Cohort Study (1905 cohort) (Nybo et al., 2001). The study was initiated in 1998, when participants were 92.2-93.8 years of age (mean age 93.2). Survivors were re-assessed in 2000, 2003 and 2005. Vital status was followed until death or January 1st 2010, whichever came first, resulting in a mean follow-up time for survivors of 11.4 years (range: 11.2-11.6). Survival status information was retrieved from the Danish Central Person Register, which is continuously updated (Pedersen et al., 2006). The younger control group (46.0-55.0 years of age, mean age 50.5) was 800 individuals from The Study of Middle Aged Danish Twins (MADT) (Skytthe et al., 2002). MADT was initiated in 1998 by random selection of 2640 intact twin pairs from 22 consecutive birth years (1931-1952) via the Danish Central Person Register. Only one twin from each twin pair was included in the present study. Permission to collect blood samples and usage of register based information was granted by The Danish National Committee on Biomedical Research Ethics for both surveys. All participants gave their informed consent.

After data cleaning, the final sample size for data analysis was 1089 oldest-old and 736 middle-aged individuals. Of these 1089 oldest-old, 14 were alive by January 1st 2010, hence, for the longitudinal analysis their remaining life expectancies were imputed using www.mortality.org, which, by appliance of data from the Statistics Denmark, contains cohort mortality data for the Danish population. Of the 736 MADT individuals, 35 had died since 1998. Based on period life table data for the Danish population (www.mortality.org) it is anticipated that only approximately $9 \%$ of the remaining 701 individuals will become 93 years of age.

\subsubsection{Replication samples}

For replication of findings from the Danish case-control study, two German samples of 1613 unrelated long-lived individuals (age 95-110) and 1104 middle-aged controls (mean age 67.2) were used. The participants were identified via the local registry offices and the Federal Administrative Office. They were recruited from different geographic regions of Germany and were all of German ancestry (Nebel et al., 2005). Approval was received from the Ethics Committee of the University Hospital Schleswig-Holstein and from local data protection authorities. All participants gave their informed consent.

For replication of findings from the Danish longitudinal study, the Dutch Leiden 85-plus Study was used. This prospective cohort was initiated in 1997, when all 85-year-old inhabitants of the city of Leiden in The Netherlands were invited to participate. The sample used here consists of 563 participants, all Caucasians and members of the 1912-1914 birth cohort recruited from 1997 to 1999 (Bootsma-van der Wiel et al., 2002). Mean follow up time for survivors is 10.35 years (range 9-11 years). The Medical Ethical Committee of the Leiden University Medical Centre approved the study. All participants gave their informed consent.

\subsection{Selection of candidate genes}

A comprehensive literature and data base search was conducted for the identification of longevity candidate genes in the GH/IGF-1/ INS signaling, DNA damage signaling and repair and pro/antioxidant pathways. The following databases were used: www.ncbi.nlm.nih. gov (/sites/entrez,/OMIM,/sites/entrez?Db= gap), www.genecards. org, www.hgvbaseg2p.org, genomics.senescence.info and genetic associationdb.nih.gov using the search terms 'human longevity', 
'human aging', 'age related disease' (including specific diseases e.g. myocardial infarction) and 'premature aging syndromes'. Additionally, genes previously identified through animal models were considered. In order to cover the core biological functions of the three candidate pathways, the needed genes were chosen using the www.ncbi.nlm. nih.gov/sites/entrez, www.biocarta.com, www.reactome.org, www. genome.utah.edu/genesnps and the www.genome.jp/kegg/pathway. html databases. In total 152 genes composing the three candidate pathways were identified, 148 of which remained after cleaning of the GoldenGate genotyping data (see below). The 148 genes, divided by pathway, are listed in Supplementary Table 1.

\subsection{Selection of SNPS}

The chromosomal regions composing the 152 candidate genes were identified through the www.ncbi.nlm.nih.gov (/gene and /mapview) and genome.ucsc.edu/databases, using the same gene ID, Sequence Accession IDs, NCBI Reference Sequence, assembly build 36 and SNP build 129. If more than one isoform of a gene transcript existed, the longest one was chosen. For each gene, 5000 bp upstream and 1000 bp downstream were included. If another gene was located within these regions, only the region until the neighboring gene was included. Due to the size of the genotyping platform, the large intron 2 of IGF1R and the large intron 2 of INSR were excluded.

Candidate SNPs were identified based on the following criteria: SNPs previously identified in genetic association studies, coding SNPs and SNPs having potential functional impact (coding nonsynonymous SNPs, SNPs located in potential splice sites or transcription factor binding sites and SNPs potentially inducing frame shifts or nonsense-mediated mRNA decay). For the identification of potential functional SNPs, the www.ncbi.nlm.nih.gov/SNP, koreanbio.org/ Variome, snpper.chip.org, snps3d.org and manticore.niehs.nih.gov data bases were used. The identity of each SNP was checked by linking back to the NCBI data base.

In order to cover as much of the common genetic variation within each gene region as possible, a number of tagging SNPs in each gene region were added, obtained through the HapMap consortium database (http://hapmap.ncbi.nlm.nih.gov/index.html.en) for the CEU cohort, using the HapMap Data Rel 23a/phase II Mar08, on NCBI B36 assembly, dbSNP build 126 criteria. Genotype data were analyzed using the HaploView software (http://www.broadinstitute.org/haploview/haploview and Barrett et al., 2005), by applying the 'pair wise tagging only', $r^{2}>0.8, \mathrm{LOD}=3$, minor allele frequency $(\mathrm{MAF})>5 \%$, and a minimum distance between SNPs $=60 \mathrm{bp}$ criteria. Finally, for optimizing the

\section{Table 1}

Characteristics of (A) the Danish discovery cohorts and (B) the German and Dutch replication samples.

\begin{tabular}{|c|c|c|c|c|}
\hline \multicolumn{2}{|l|}{ (A) The Danish discovery cohorts } & \multicolumn{2}{|c|}{ MADT } & 1905 \\
\hline \multicolumn{2}{|l|}{ Number of individuals } & \multicolumn{2}{|c|}{736} & 1089 \\
\hline \multicolumn{2}{|c|}{ Average age in years at intake (range) } & \multicolumn{2}{|c|}{$50.6(46.0-55.0)$} & $93.2(92.2-93.8)$ \\
\hline \multicolumn{2}{|c|}{ Male:female ratio (\%) at intake } & \multicolumn{2}{|c|}{$50.4: 49.6$} & $28.7: 71.3$ \\
\hline \multicolumn{2}{|l|}{ Mean follow up time (SD) } & \multicolumn{2}{|l|}{ - } & $3.53(2.5)$ \\
\hline \multicolumn{2}{|l|}{ Follow up time (years) } & \multicolumn{2}{|l|}{-} & 11.4 \\
\hline \multirow[t]{2}{*}{ (B) The replication samples } & \multicolumn{3}{|c|}{$\begin{array}{l}\text { German case-control } \\
\text { study }\end{array}$} & $\begin{array}{l}\text { Dutch longitudinal } \\
\text { study }\end{array}$ \\
\hline & \multicolumn{3}{|c|}{ Controls } & $\begin{array}{l}\text { Leiden 85-plus } \\
\text { Study }\end{array}$ \\
\hline Number of individuals & 110 & & 1613 & 563 \\
\hline Average age at intake (SD) & 67.2 & & $98.4(2.7)$ & $85.0(0)$ \\
\hline Male:female ratio (\%) at intake & 25.6 & & $26.8: 73.2$ & $33.4: 66.6$ \\
\hline Mean follow up time (SD) & - & & - & $5.49(3.0)$ \\
\hline Follow up time (years) & - & & - & 10.4 \\
\hline
\end{tabular}

Notes: MADT: the Study of Middle Aged Danish Twins, 1905: the Danish 1905 Birth cohort, LLI: long lived individuals, SD: standard deviation. quality of the genotype data, SNPs known to perform poorly on the Illumina GoldenGate genotyping platform were excluded. In total, 1415 SNPs were chosen and 1273 were successfully genotyped.

\subsection{Genotyping and data cleaning}

DNA was isolated from blood spot cards using the QIAamp DNA Mini and Micro Kits (Qiagen, Düsseldorf, Germany). The genotyping was conducted using the Illumina GoldenGate platform (Illumina Inc, San Diego, CA, USA) and data cleaning was carried out according to the manufacturer's recommendations using the GenomeStudio software (http:// www.illumina.com/software/genomestudio_software.ilmn); primary exclusion criteria were sample call rate $<90 \%$ and SNP call frequency $<$ $90 \%$. Subsequently, SNPs clustering in the following categories were manually evaluated: a call frequency $90-95 \%$, close SNP clusters ( score $<2.3$ ), clusters with low intensity (score $<0.2$ or $>0.8$ ), cluster having a heterozygote cluster shifting towards a homozygote cluster (score $<0.13$ ), SNPs with excess heterozygosity (score $<-0.3$ or $>0.2$ ) and SNPs located on the X chromosome (considering male hemizygosity). In total, 142 SNPs and 175 DNA samples were excluded leaving 1825 individuals, 148 candidate genes and 1273 SNPs for data analysis. Furthermore, 24 samples were included twice in order to investigate the intra-plate reproducibility and 48 samples were included twice to test the inter-plate reproducibility. Data showed an intra-plate reproducibility of $99.4 \%$ and an inter-plate reproducibility of $96.8 \%$.

Genotyping of the German replication samples were performed using either Sequenom MassARRAY iPLEX®Gold technology (Sequenom ${ }^{\circledR}$, Inc., CA, USA) with iPlex primers designed via the MassARRAY Design Software, or via an automated platform using predesigned TaqMan ${ }^{\circledR}$ SNP genotyping assays (Applied Biosystems) (Hampe et al., 2001); PCR was performed using the GeneAmp PCR System 9700 (Applied Biosystems) under standard conditions and the ABI Prism 7900HT Sequence Detection System and the Sequence Detection System Software 2.0 (Applied Biosystems) were used for allelic discrimination.

The Dutch replication samples were genotyped via Sequenom MassARRAY iPLEX®Gold technology (Sequenom®, Inc., CA, USA), using primers designed via the SpectroDESIGNER (Sequenom ${ }^{\circledR}$, Inc., CA, USA) and the high plex reaction protocol (www.sequenom.com/ iplex). Since rs207444 and rs3211994 were not compatible with the iPLEX format, genotyping was conducted using assay-on-demand TaqMan ${ }^{\circledR}$ SNP genotyping assays (Applied Biosystems); PCR was performed on the GeneAmp PCR system 9700 (Applied Biosystems, CA, USA) under universal conditions and the Applied Biosystems 7900HT Fast Real-time PCR System and SDS2.3 Software (Applied Biosystems, CA, USA) were used for allelic discrimination.

\subsection{Statistical analysis}

In the case-control study of the Danish discovery cohorts singleSNP case-control comparisons of allele frequencies (CCA) were performed using the Plink statistical program (http://pngu.mgh. harvard.edu/purcell/plink, Purcell et al., 2007) by two-tailed Chisquare test. Replication of the findings from the Danish case-control study was conducted by CCA in the German case-control samples in the same way.

Approximately 1 in 20 members of the 1905 birth cohort in Denmark had survived until 1998 (start of the Danish 1905 Birth Cohort Study) and approximately 1 in 20 of these members survived until 100 years of age, i.e. reaching age 92-93 (the inclusion age of the Danish 1905 Birth Cohort Study participants) is only "halfway to becoming a centenarian" in terms of selection. In the longitudinal survival analysis of the oldest-old from the Danish 1905 Cohort Study, two survival variables were used. First, we wanted to investigate the genetic contribution to surviving for only a short period of time as opposed to surviving for a longer period of time during the ninth 
decade of life. Since the distribution of survival times was clearly right skewed (data not shown), a cut-off with respect to time of death was made at December 31st 2000, when approximately half of the males and one third of the females in the cohort had died. Consequently, the early/late death variable was defined as: early=death occurred between 1998 (date at intake) and December 31st 2000 (i.e. individuals living to maximum age 95) and late $=$ death occurred after January 1st 2001 (i.e. individuals surviving to age $95+$ ). As the second variable, we used the number-of-days-lived defined as the exact number of days lived from date of intake in 1998 to either death or the imputed date (the latter is described in Section 2.1.1). For this variable, the assumptions of normal distribution and equal variance were tested in the STATA 11.1 statistical program (Stata Corporation, College Station, TX, USA) by Shapiro-Wilk and BreuschPagan/Cook-Weisberg tests. Since the variable did not show normal distribution, it was transformed to the square root for a better fit. Association analysis was performed by two-tailed linear (for the number-of-days-lived variable) or logistic (for the early/late death variable) regression in Plink, adjusting for sex and age or stratifying by sex, while adjusting for age.

Replication of the findings from the Danish longitudinal study was performed in Stata 11.1 by analyzing genotype data from the Leiden 85-plus Study using a sex-adjusted Cox proportional hazard model. An additive model was used, and only in cases when inspection of Kaplan-Meier plots indicated a recessive or dominant model, such a model was applied.

In order to obtain a quantitative estimate for the differences in mean survival time between individuals homozygous for the common alleles of a SNP found to be associated with the number-ofdays-lived variable and individuals being either heterozygous or homozygous for the rare allele at the same SNP, regression analysis was performed for same sex and same age (93 years) individuals and the regression coefficients were used for estimation. Moreover, the relative risks (RRs) of being alive after age 95 (corresponding to the 1 st of January 2001) were calculated for the heterozygotes and homozygotes for the rare allele of each SNP found to be associated to the early/late death variable. The RRs between genotype groups were estimated based on the ORs obtained in the logistic regression analysis. All these analyses were performed in STATA 11.1.

For all the analyses, the nominal significance level was set to 0.05 . Correction for multiple testing within each pathway was performed by a permutation approach applying $\max (\mathrm{T})$ permutation mode set at 10,000 permutations for the analyses carried out in the Plink software. Correction for multiple testing in analyses performed in the STATA software was done using the Bonferroni approach.

\section{Results}

By conducting a thorough literature and data base search candidate genes, candidate SNPs and tagging SNPs were identified (see Sections 2.2 and 2.3 for details). A total of 148 candidate genes and 1273 SNPs were successfully genotyped and are listed in Supplementary Table 1: these constitute 592 SNPs in 77 genes of the DNA damage signaling and DNA repair pathway, 370 SNPs in 33 genes of the GH/IGF-1/INS signaling pathway and 311 SNPs in 38 pro/antioxidant genes. The characteristics of the Danish discovery cohorts of middleaged and oldest-old individuals are described in Table $1 \mathrm{~A}$, while the German and Dutch replication samples are described in Table 1B.

\subsection{Case-control comparison of middle-aged and oldest-old Danes}

Exploiting the case-control allele comparisons revealed considerably more nominally significant differences than would be expected by chance (see Supplementary Table 2). After adjusting allelic comparisons for multiple testing by permutation, 10 of the 1273 SNPs remained significant; of these SNPs the minor allele of the GH/IGF-
1/INS-SNPs rs1207362 (KL), rs2267723 (GHRHR) and rs572169 (GHSR), as well as the pro/antioxidant-SNP rs1002149 (GSR) and the DNA-repair-SNP rs1805329 (RAD23B) decreased in frequency from middle-aged to old-aged ( $\mathrm{OR}<1$ ), whereas the GH/IGF-1/INSSNPs rs3842755 (INS) and rs9456497 (IGF2R) and the DNA-repairSNPs rs11571461 (RAD52), rs13251813 (WRN) and rs2953983 $(P O L B)$ increased in frequency $(\mathrm{OR}>1)$. As sex-specific differences previously have been suggested for association of SNPs with longevity (Candore et al., 2006; Franceschi et al., 2000; Li et al., 2009; Soerensen et al., 2010) a sex-stratified analysis was also performed; the analysis supported the associations of the 10 SNPs mentioned above for both genders (data not shown), and in addition identified 1 DNA-repairSNP in males; the minor allele of rs3211994 (NTLH1) increased in frequency from middle-aged to old-aged. The data are summarized in Table 2 .

The 11 SNPs were subsequently analyzed in replication samples of 1613 long-lived and 1104 middle-aged Germans. The MAF of the SNPs in the German and Danish case-control samples are listed in Supplementary Table 3. None of the 11 SNPs formally replicated in the German samples (see Table 3), although rs11571461 (RAD52) did show a non-significant tendency of association in the same direction $(95 \% \mathrm{CI}(\mathrm{OR})=[0.927-1.457])$.

\subsection{Longitudinal study in the cohort of oldest-old Danes}

As for the case-control study, analysis of survival data in the oldest-old Danes revealed more nominally significant associations than would be expected by chance, whether using the categorical early/late death or the numeric number-of-days-lived variables (see Supplementary Table 4). After correcting for multiple testing 2 SNPs were found to be associated with late life survival for both genders combined: carriers of the rare allele of the pro/antioxidant-SNP rs10047589 (TXNRD1) displayed a significant increase in survival when categorizing into early/late death, whereas the rare allele of the pro/antioxidant-SNP rs207444 $(X D H)$ was found to be significantly associated with increased number of days lived (see Table 4A). When stratifying by sex, these results were supported in both genders (data not shown). Moreover, when applying the early/late death variable, positive effects of the rare alleles of the GH/IGF-1/ INS-SNP rs26802 (GHRL) and the DNA-repair-SNP rs13320360 (MLH1) on survival were observed in males, while the DNA-repairSNP rs2509049 (H2AFX) had a negative effect on survival in females. Using number-of-days-lived as the measure of survival, the rare allele of the DNA-repair-SNP rs705649 (XRCC5) positively affected survival in males. The results are summarized in Table 4B.

Since we performed the longitudinal analyses as regression analyses, we were able to estimate the quantitative effects of the 6 SNPs, represented by the risk differences (RD) for the SNPs associated with the early/late death variable, and by the additional survival time for the SNPs associated with the number-of-days-lived variable. These estimates indicated rather substantial effects: a maximum RD was seen for rs26802 (GHRL) CC males having a 53\% higher chance for being alive after age 95 (corresponding to the 1st of January 2001) as compared to AA homozygotes, while a maximum difference of 1.66 additional years of survival was seen for rs705649 (XRCC5) GG males as compared to rs705649-AA males, who obtained a mean survival time of 2.15 years. The data are summarized in Supplementary Tables $5 \mathrm{~A}$ and $\mathrm{B}$.

Next the 6 SNPs were evaluated in a replication cohort of $56385+$ year old Dutch individuals. The frequency data of the 6 SNPs in the Dutch and Danish longitudinal cohorts are listed in Supplementary Table 6. Applying an additive model, none of the SNP associations was replicated in the Dutch cohort (see Table 5). However, inspection of Kaplan-Meier survival plots for the 6 SNPs suggested a recessive mode of effect for rs10047589 (TNXRD1) in the Dutch data (data not shown), with a similar direction of effect as in the Danish 
Table 2

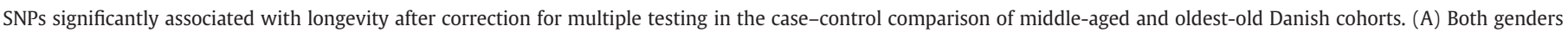
combined and (B) additional SNP in sex-stratified analyses.

\begin{tabular}{|c|c|c|c|c|c|c|c|c|c|}
\hline & Gene & SNP & $\begin{array}{l}\text { Minor/major } \\
\text { allele }\end{array}$ & Chr./position & $\begin{array}{l}\text { Position in } \\
\text { gene }\end{array}$ & MAF MADT/1905 & $\begin{array}{l}\text { PCCA } \\
\text { uncorrected }\end{array}$ & $\begin{array}{l}\text { PCCA } \\
\text { corrected }\end{array}$ & OR $(95 \% \mathrm{CI})$ \\
\hline \multicolumn{10}{|l|}{ (A) Both genders } \\
\hline Pro/antioxidants & GSR & rs1002149 & $\mathrm{A} / \mathrm{C}$ & $8 / 30705280$ & $5^{\prime}$ & $0.183 / 0.137$ & $1.47 \cdot 10^{-4}$ & 0.0335 & $0.706(0.589-0.845)$ \\
\hline \multirow[t]{5}{*}{$\mathrm{GH} / \mathrm{IGF} / \mathrm{INS}$} & INS & rs3842755 & $\mathrm{A} / \mathrm{C}$ & $11 / 2137372$ & 3' UTR & $0.292 / 0.424$ & $8.38 \cdot 10^{-16}$ & $1 \cdot 10^{-4}$ & $1.789(1.552-2.063)$ \\
\hline & $\mathrm{KL}$ & rs1207362 & $\mathrm{A} / \mathrm{C}$ & $13 / 32510839$ & Intronic & $0.339 / 0.245$ & $2.77 \cdot 10^{-9}$ & $1 \cdot 10^{-4}$ & $0.634(0.545-0.737)$ \\
\hline & GHRHR & rs2267723 & $\mathrm{G} / \mathrm{A}$ & 7/30973467 & Intronic & $0.414 / 0.330$ & $2.28 \cdot 10^{-7}$ & $1 \cdot 10^{-4}$ & $0.696(0.607-0.799)$ \\
\hline & GHSR & rs572169 & $\mathrm{A} / \mathrm{G}$ & $3 / 173648421$ & Coding (syn) & $0.342 / 0.274$ & $1.65 \cdot 10^{-5}$ & 0.0045 & $0.725(0.626-0.839)$ \\
\hline & IGF2R & rs9456497 & $\mathrm{G} / \mathrm{A}$ & $6 / 160363418$ & Intronic & $0.191 / 0.252$ & $1.86 \cdot 10^{-5}$ & 0.0050 & $1.424(1.211-1.674)$ \\
\hline \multirow[t]{4}{*}{ DNA repair } & RAD52 & rs11571461 & $\mathrm{G} / \mathrm{A}$ & $12 / 896580$ & Intronic & $0.098 / 0.195$ & $5.05 \cdot 10^{-15}$ & $1 \cdot 10^{-4}$ & $2.226(1.815-2.730)$ \\
\hline & WRN & rs13251813 & $\mathrm{A} / \mathrm{G}$ & $8 / 31083753$ & Intronic & $0.077 / 0.134$ & $1.72 \cdot 10^{-7}$ & $2 \cdot 10^{-4}$ & $1.840(1.460-2.318)$ \\
\hline & POLB & rs2953983 & $\mathrm{G} / \mathrm{A}$ & $8 / 42332413$ & Intronic & $0.080 / 0.123$ & $4.54 \cdot 10^{-5}$ & 0.0225 & $1.599(1.274-2.006)$ \\
\hline & RAD23B & rs1805329 & $\mathrm{A} / \mathrm{G}$ & $9 / 109124149$ & Coding (non-syn) & $0.245 / 0.190$ & $6.74 \cdot 10^{-5}$ & 0.0309 & $0.722(0.615-0.847)$ \\
\hline \multicolumn{10}{|l|}{ (B) Males } \\
\hline DNA repair & NTLH1 & rs3211994 & $\mathrm{A} / \mathrm{G}$ & $16 / 2029029$ & $3^{\prime}$ & $0.112 / 0.201$ & $1.38 \cdot 10^{-5}$ & 0.0056 & $1.994(1.455-2.732)$ \\
\hline
\end{tabular}

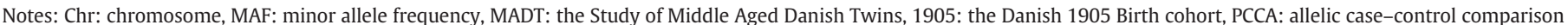
P-value, OR: odds ratio, 95\% CI: 95\% confidence interval, UTR: untranslated region, Syn: synonymous, Non-syn: Non-synonymous.

longitudinal study, although the association was not significant; $\mathrm{HR}=0.758, \mathrm{p}=0.103,95 \%, \mathrm{CI}=[0.543-1.058]$.

\subsection{Similar directions of effect in the Danish case-control and longitudinal studies}

We wanted to explore whether the 11 SNPs identified via the Danish case-control study maintained an effect during old age. Consequently we investigated the 11 SNPs in the longitudinal data on the oldest-old Danes. Similar directions of effects, i.e. positive effects on survival, were noted for 5 SNPs; when applying the number-of-days-lived variable, rs13251813 (WRN) and rs3842755 (INS) displayed nominally significant associations $(\mathrm{p}<0.05)$, the former in the entire group of oldest-old and the latter when restricting to males. Additionally, rs9456497 (IGF2R) showed a borderline significant association when restricting to males $(p=0.05)$. For the early/late death variable, rs3211994 (NTHL1) displayed nominal significance in both genders combined, while rs1157146 (RAD52) showed a non-significant tendency in females only $(\mathrm{p}=0.10)$. None of the remaining six SNPs showed similar directions of effect. The data is summarized in Table 6A.

Finally, in order to explore whether the 6 SNPs, identified in the longitudinal study of oldest-old Danes, presented a similar direction of effects on survival from middle age to old age, we investigated the 6 SNPs in the Danish case-control data. Only rs207444 (XDH) displayed a nominally significant effect in the same direction as found in the oldest old $(\mathrm{p}=0.005)$. The data is summarized in Table 6B.

\section{Discussion}

The vast majority of the candidate genetic association studies of longevity published so far has explored only a small number of variations and/or genes. The investigation of large collections of candidate variations grouped together based on the functional roles of the gene products is a much more recent strategy (Flachsbart et al., 2010; Nebel et al., 2009; Pawlikowska et al., 2009b). In this study we investigated 592 SNPs in 77 genes of the DNA damage signaling and DNA repair pathway, 370 SNPs in 33 genes of the GH/IGF-1/INS signaling pathway and 311 SNPs in 38 pro/antioxidant genes, making it the most comprehensive study to date following this strategy. Furthermore, in addition to the widely used case-control approach, we also present data from a longitudinal analysis of survival in the oldest-old.

In the analysis of the case-control study of 1089 oldest-old and 736 middle-aged Danes, we found 11 novel SNPs in 11 different genes to be associated with human longevity. The rare allele of variants in INS, IGF2R, POLB, RAD52, WRN and NTHL1 was demonstrated to be advantageous, the latter most evident in males, whereas the rare allele of variants in KL, GHRHR, GHSR, GSR, and RAD23B was disadvantageous for survival.

None of the findings could be formally confirmed in a German replication cohort of comparable size, although rs11571461 (RAD52) did show a tendency in the same direction as found in the Danish data. In support of our findings, other variants in KL, WRN and NTHL1 have previously been associated with longevity (Arking et al., 2005; Castro et al., 2000; Yashin et al., 2010) and rs687045 (KL) which was put forward by Lunetta et al. as a mortality SNP during old age (Lunetta et al., 2007), is in our cohorts in moderate $L D\left(R^{2}=0.25\right)$ with rs1207362 $(K L)$ described in our study as disadvantageous for longevity. Moreover, supportive of an association of INS, a very recent publication of gene-based case-control analysis of genes in the IGF-1/ Insulin pathway reported gene-based association of INS with longevity (Deelen et al., 2011). Additionally, some of the 11 SNPs have been

Table 3

Replication of Danish case-control findings in the German case-control samples.

\begin{tabular}{|c|c|c|c|c|c|c|}
\hline & Gene & SNP & OR (Danish study) & OR (German study) & PCCA Germans & $95 \% \mathrm{CI}$ \\
\hline Pro/antioxidants & GSR & rs1002149 & 0.706 & 1.054 & 0.470 & $0.913-1.217$ \\
\hline \multirow[t]{5}{*}{$\mathrm{GH} / \mathrm{IGF} / \mathrm{INS}$} & INS & rs3842755 & 1.789 & 0.949 & 0.434 & $0.832-1.082$ \\
\hline & $\mathrm{KL}$ & rs1207362 & 0.634 & 0.985 & 0.804 & $0.873-1.110$ \\
\hline & GHRHR & rs2267723 & 0.696 & 1.005 & 0.934 & $0.900-1.121$ \\
\hline & GHSR & rs572169 & 0.725 & 1.106 & 0.097 & $0.981-1.247$ \\
\hline & IGF2R & rs9456497 & 1.424 & 0.962 & 0.586 & 0.837-1.105 \\
\hline \multirow[t]{4}{*}{ DNA repair } & RAD52 & rs11571461 & 2.226 & 1.162 & 0.191 & $0.927-1.457$ \\
\hline & WRN & rs13251813 & 1.84 & 0.999 & 0.998 & $0.754-1.325$ \\
\hline & POLB & rs2953983 & 1.599 & 1.046 & 0.683 & $0.842-1.299$ \\
\hline & RAD23B & rs1805329 & 0.722 & 0.955 & 0.507 & $0.833-1.094$ \\
\hline DNA repair (males) & NTHL1 & rs3211994 & 1.994 & 0.931 & 0.627 & $0.699-1.241$ \\
\hline
\end{tabular}

Notes: OR: odds ratio, PCCA: allelic case-control comparison P-value, 95\% CI: 95\% confidence interval. 


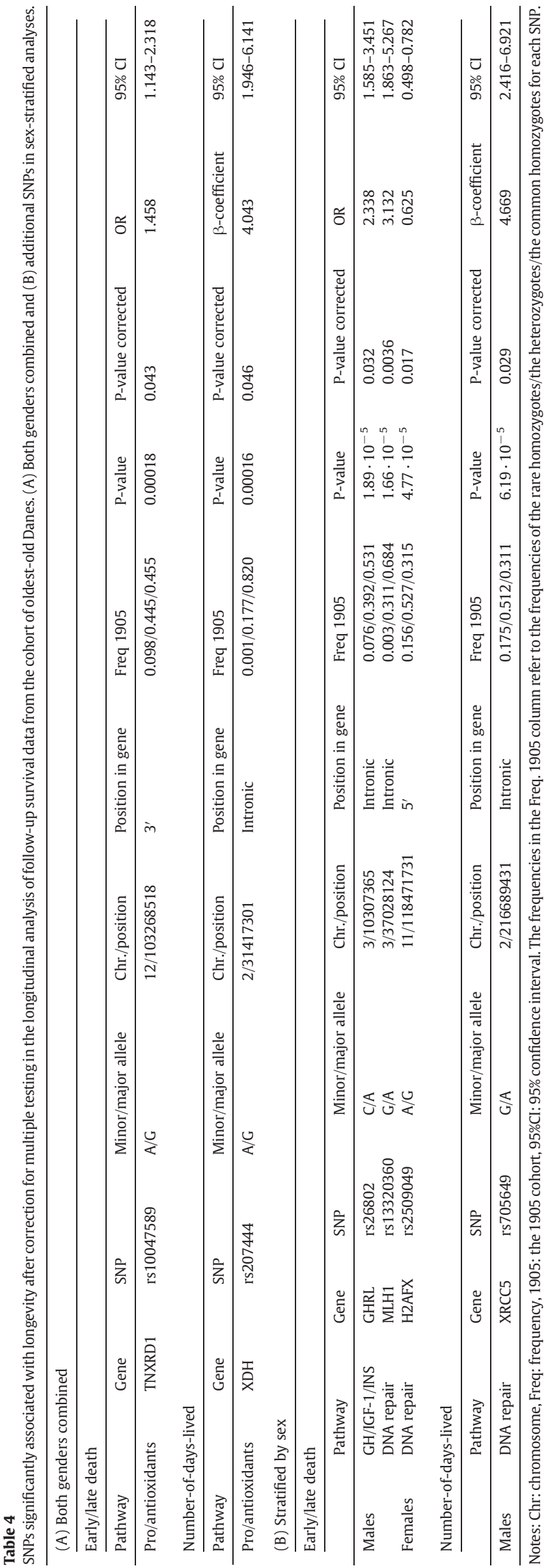

reported as associated with phenotypes of possible relevance to variation in lifespan; rs572169 (GHSR) with obesity (Gueorguiev et al., 2009), and rs2953983 (POLB), rs1805329 (RAD23), rs11571461 (RAD52) and rs13251813 (WRN) with different types of cancers (Pan et al., 2009; Schildkraut et al., 2010; Van Ness et al., 2008; Yang et al., 2009).

An interesting question was whether the genetic variants with a proposed effect on survival from middle age to old age would also demonstrate an effect for survival during old age. We therefore tested the 11 SNPs for association to survival in the Danish 1905 cohort using the longitudinal design and found 3 SNPs to display the same nominal significant $(p<0.05)$ direction of effect in the longitudinal data, while 2 SNPs showed similar tendencies $(0.05<\mathrm{p}<0.10)$. Interestingly, all 5 SNPs were apparent longevity SNPs, i.e. SNPs with a positive effect on survival. This is in line with a recent study suggesting that long life is not due to a decreased burden of negatively affecting disease variants but rather due to positively influencing longevity variants (Beekman et al., 2010).

A lack of similar effects in the longitudinal data for the remaining SNPs could indicate that these SNPs may be relevant at different ages, i.e. the influence of genes and gene variants may be of consequence for survival in young elderly, but lose this importance at the highest ages.

When investigating the association of the 1273 SNPs with survival at advanced age using a longitudinal study approach, we identified 6 novel SNPs in 6 genes to be associated with longevity. The rare allele of a variant in H2AFX appeared disadvantageous, while the rare variants in GHRL, MLH1, TXNRD1, XDH and XRCC5 were advantageous for longevity. In line with these results an MLH1 haplotype was previously reported to be associated with longevity in a case-control study (Kim et al., 2006), whereas genetic variants in GHRL have been described as associated with phenotypes of possible relevance to variation in lifespan; high HDL-cholesterol levels (Hubacek et al., 2007) and hypertension (Berthold et al., 2010). Finally, the activity of the XRCC5 protein product Ku80 has been reported to decrease from young to old age in humans (Scarpaci et al., 2003), indicating a biological change with age.

The Danish case-control study supported an advantageous effect of rs207444 $(X D H)$ also on survival from middle age to old age, and additionally some support was observed for rs10047589 (TNXRD1) in the Dutch replication cohort, indicating a recessive mode of effect for this variant.

Interestingly, the longitudinal study revealed more longevityassociated SNPs in the sex-stratified analysis (4 of 6 SNPs) than the Danish case-control study ( 1 of 11 SNPs). This observation could indicate that the impact of genetic effect becomes more genderspecific during advanced age than at younger ages, and that the genetic effect in old age might be most pronounced for males. Such a difference between genders was recently suggested by Montesanto et al. (2011) for Italians.

From a pathway perspective a few comments can be made on the genes holding the SNPs suggested here. For the GH/IGF-1/INS pathway the present data suggests that variations in genes whose products take part in growth hormone synthesis and secretion (GHRHR, GHRL and GHSR) or in signaling to and affecting target organs/cells (INS, KL, IGF2R) might pose effects on longevity. With respect to DNA repair, the gene products of the genes holding the significant SNPs take part in different DNA repair activities, though the majority take part in base excision repair (NTHL1, POLB, and WRN) or in DNA double strand break repair (RAD52, H2AFX, XRCC5 and WRN), thus indicating that variation in the repair of DNA damage induced by for instance reactive oxygen species and ionizing radiation could pose effects on longevity. Lastly, with respect to the pro/antioxidant pathway both antioxidants (GSR and TXNRD1) and a prooxidant $(X D H)$ are observed, indicating that the role of this pathway in longevity appears complex. 
Table 5

Replication of findings from the Danish longitudinal study in the longitudinal Dutch Leiden 85-plus Study.

\begin{tabular}{|c|c|c|c|c|c|c|}
\hline \multicolumn{3}{|c|}{ Both genders combined } & \multirow{2}{*}{$\frac{\text { Danish longitudinal study }}{\text { Estimate }}$} & \multicolumn{3}{|c|}{ Dutch longitudinal study } \\
\hline Pathway & Gene & SNP & & HR & P-value & $95 \% \mathrm{CI}$ \\
\hline Pro/antioxidants & TNXRD1 & rs10047589 & $1.458(\mathrm{OR})$ & 0.969 & 0.659 & $0.844-1.112$ \\
\hline Pro/antioxidants & $\mathrm{XDH}$ & rs207444 & $4.043(\beta)$ & 0.886 & 0.404 & $0.667-1.177$ \\
\hline \multicolumn{7}{|l|}{ Males } \\
\hline GH/IGF-1/INS & GHRL & rs26802 & 2.338 (OR) & 0.730 & 0.962 & 0.774-1.197 \\
\hline \multirow[t]{2}{*}{ DNA repair } & MLH1 & rs13320360 & 3.132 (OR) & 1.169 & 0.827 & $0.288-4.727$ \\
\hline & XRCC5 & rs705649 & $4.669(\beta)$ & 1.037 & 0.749 & $0.828-1.300$ \\
\hline \multicolumn{7}{|l|}{ Females } \\
\hline DNA repair & H2AFX & rs2509049 & $0.625(\mathrm{OR})$ & 0.909 & 0.260 & $0.769-1.073$ \\
\hline
\end{tabular}

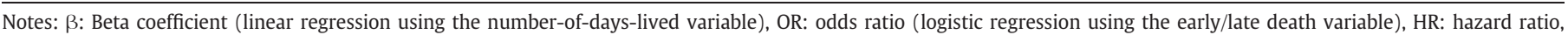
95\% CI: 95\% confidence interval.

The SNPs suggested here to associate to longevity might induce different functional effects; rs1002149 (GSR) and rs2509049 (H2AFX) located $5^{\prime}$ of genes might affect the level of gene expression, while rs3842755 (INS), rs3211994 (NTHL1) and rs10047589 (TNXRD1) are all located $3^{\prime}$ and could thus in principle influence decay of mRNA. The non-synonymous coding SNP rs1805329 (RAD23B) could potentially affect protein activity, while the intronic SNPs (rs1207362 (KL), rs2267723 (GHRHR), rs9456497 (IGF2R), rs11571461 (RAD52), rs13251813 (WRN), rs2953983 (POLB), rs207444 (XDH), rs26802 (GHRL), rs13320360 (MLH1) and rs705649 (XRCC5)) could possibly induce splicing alterations. Alternatively, the associated SNPs might simply be proxies for other truly causative variants.

How such suggested functional effects might impact aging and/or longevity is an extremely difficult question, especially considering that the role of the three pathways in aging and/or longevity appears very complex. In any case, functional studies of the molecular effects of the associated variants are needed

Due to the emergence of high throughput techniques facilitating studies of sizeable numbers of variations, the replication of initial findings has become a major issue in genetic epidemiology. In this study we tested our findings from the case-control and the longitudinal study using German samples of 1613 long-lived individuals (95+) and 1104 middle-aged controls, and a cohort of 85 + Dutch elderly, respectively. No formal replication was observed in either case although a few supportive observations were found (see above).
A number of reasons can be given for the lack of true replication. It could be a matter of under-powered replication populations. However, the number of German samples is larger than the Danish discovery cohorts, thus arguing against this explanation. Alternatively it might be due to heterogeneity between samples, for instance differences in enrollment of the participants. As seen in Table 1, the German cases are older than the Danish oldest-old, whereas the members of the Dutch $85+$ cohort are somewhat younger at intake than the Danish 1905 cohort members. Hence, if the effects of the variants are not constant throughout old age, they may not replicate well in these study samples. Change in association effects with age has been reported previously (Jacobsen et al., 2010). Finally, it might simply be that the associations are population specific, perhaps mediated through population specific characteristics in environment. Such population specific effects have previously been suggested (Di Bona et al., 2009).

In our study we only performed correction of multiple testing within each pathway, since the selection of genes and SNPs was based on a sound a priori hypothesis. Moreover, due to the design of the statistical software, we corrected for multiple testing within each type of test, and hence, we did not correct for performing the sex-stratified analysis as well as a test for both genders combined, and with respect to the longitudinal study for investigating the 2 survival variables. Such corrections should in principle be included, in order to avoid false positive findings. If correcting the p-values from

Table 6

Similar directions of effect in the Danish case-control and longitudinal studies.

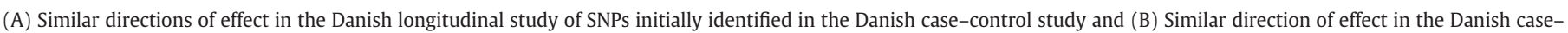
control study of a SNP initially identified in the Danish longitudinal study.

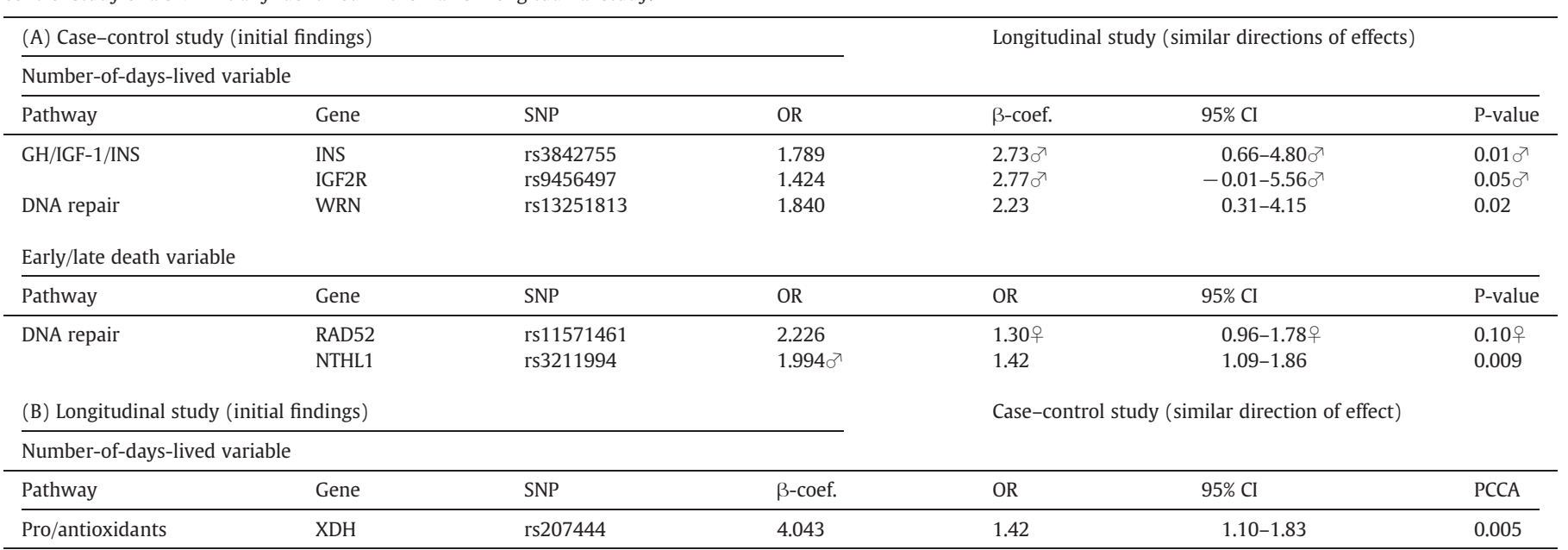

Notes: OR: odds ratio, $\beta$-coef: beta coefficient, 96\% CI: 95\% confidence interval, ơ : males separately, 우 females separately, PCCA: allelic case-control comparison P-value. 
each pathway using the overly conservative Bonferroni approach 8 of the 11 SNPs from the case-control study remained significant, while 1 of the 6 SNPs identified in the longitudinal study remained significant. By contrast, correction by the less conservative Benjamini and Hochberg (1995) method, left more associations significant (10 SNPs from the case-control study and 1 SNP from the longitudinal study), thus underscoring the difference in conclusions based on choice of method of correction. In any case, both of these correction methods assume independency between the tests, which is clearly not the case in our study.

In conclusion we here present new candidate variations for human longevity located in the DNA damage signaling and DNA repair, the GH/IGF-1/INS signaling and pro/antioxidant pathways. We find that 4 of the 17 identified SNP associations show significant and similar direction of effect when using the two different study approaches applied to the Danish discovery cohorts, while 2 SNP associations showed similar tendencies. However, none of the 17 SNP associations displayed replication in the independent samples from Germany and the Netherlands, although for 2 SNP associations some support was noticed.

\section{Conflicts of interest}

\section{The authors have no conflicts to declare.}

\section{Acknowledgments}

This study was supported by the Max-Planck Institute for Demographic Research (Rostock, Germany), the INTERREG 4 A programme Syddanmark-Schleswig-K.E.R.N (by EU funds from the European Regional Development Fund), the National Institute on Aging (P01 AG08761), a Dutch grant from the Netherlands Consortium for Healthy Aging (Grant 050-060-810) in the framework of the Netherlands Genomics Initiative/Netherlands Organization for Scientific Research (NWO), the European Union's Seventh Framework Programme (FP7/2007-2011) under grant agreement $n^{\circ} 259679$, the Novo Nordisk Foundation, the Aase and Ejnar Danielsen Foundation, the Augustinus Foundation, the Brødrene Hartmann Foundation, the King Christian the 10th Foundation and the Einer Willumsens Mindelegat Foundation. The Danish Aging Research Center is supported by a grant from the VELUX Foundation. Susanne Knudsen, Steen Gregersen, Ulla Munk, Shuxia Li, Anne Mette Hedegaard Nielsen, Marlene Graff Sørensen and Lene Elnegaard are thanked for excellent technical work.

\section{Appendix A. Supplementary data}

Supplementary data to this article can be found online at doi:10. 1016/j.exger.2012.02.010.

\section{References}

Arking, D.E., Atzmon, G., Arking, A., Barzilai, N., Dietz, H.C., 2005. Association between a functional variant of the KLOTHO gene and high-density lipoprotein cholesterol, blood pressure, stroke, and longevity. Circ. Res. 96, 412-418.

Barrett, J.C., Fry, B., Maller, J., Daly, M.J., 2005. Haploview: analysis and visualization of LD and haplotype maps. Bioinformatics 21, 263-265.

Bartke, A., 2005. Minireview: role of the growth hormone/insulin-like growth factor system in mammalian aging. Endocrinology 146, 3718-3723.

Beekman, M., Nederstigt, C., Suchiman, H.E., Kremer, D., van der, B.R., Lakenberg, N., Alemayehu, W.G., de Craen, A.J., Westendorp, R.G., Boomsma, D.I., de Geus, E.J., Houwing-Duistermaat, J.J., Heijmans, B.T., Slagboom, P.E., 2010. Genome-wide association study (GWAS)-identified disease risk alleles do not compromise human longevity. Proc. Natl. Acad. Sci. U. S. A. 107, 18046-18049.

Benjamini, Y., Hochberg, Y., 1995. Controlling the false discovery rate: a practical and powerful approach to multiple testing. J. R. Stat. Soc. B 57, 289-300.

Berthold, H.K., Giannakidou, E., Krone, W., Tregouet, D.A., Gouni-Berthold, I., 2010. Influence of ghrelin gene polymorphisms on hypertension and atherosclerotic disease. Hypertens. Res. 33, 155-160.
Bonafe, M., Barbieri, M., Marchegiani, F., Olivieri, F., Ragno, E., Giampieri, C., Mugianesi, E., Centurelli, M., Franceschi, C., Paolisso, G., 2003. Polymorphic variants of insulinlike growth factor I (IGF-I) receptor and phosphoinositide 3-kinase genes affect IGF-I plasma levels and human longevity: cues for an evolutionarily conserved mechanism of life span control. J. Clin. Endocrinol. Metab. 88, 3299-3304.

Bootsma-van der Wiel, A., Van Exel, E., de Craen, A.J., Gussekloo, J., Lagaay, A.M., Knook, D.L., Westendorp, R.G., 2002. A high response is not essential to prevent selection bias: results from the Leiden 85-plus study. J. Clin. Epidemiol. 55, 1119-1125.

Burtner, C.R., Kennedy, B.K., 2010. Progeria syndromes and ageing: what is the connection? Nat. Rev. Mol. Cell Biol. 11, 567-578.

Cabelof, D.C., Ikeno, Y., Nyska, A., Busuttil, R.A., Anyangwe, N., Vijg, J., Matherly, L.H. Tucker, J.D., Wilson, S.H., Richardson, A., Heydari, A.R., 2006. Haploinsufficiency in DNA polymerase beta increases cancer risk with age and alters mortality rate. Cancer Res. 66, 7460-7465.

Candore, G., Balistreri, C.R., Listi, F., Grimaldi, M.P., Vasto, S., Colonna-Romano, G., Franceschi, C., Lio, D., Caselli, G., Caruso, C., 2006. Immunogenetics, gender, and longevity. Ann. N. Y. Acad. Sci. 1089, 516-537.

Castro, E., Edland, S.D., Lee, L., Ogburn, C.E., Deeb, S.S., Brown, G., Panduro, A., Riestra, R. Tilvis, R., Louhija, J., Penttinen, R., Erkkola, R., Wang, L., Martin, G.M., Oshima, J., 2000. Polymorphisms at the Werner locus: II. 1074Leu/Phe, 1367Cys/Arg, longevity, and atherosclerosis. Am. J. Med. Genet. 95, 374-380.

Christensen, K., Johnson, T.E., Vaupel, J.W., 2006. The quest for genetic determinants of human longevity: challenges and insights. Nat. Rev. Genet. 7, 436-448.

Christiansen, L., Bathum, L., Frederiksen, H., Christensen, K., 2004. Paraoxonase 1 polymorphisms and survival. Eur. J. Hum. Genet. 12, 843-847.

Cluett, C., Melzer, D., 2009. Human genetic variations: beacons on the pathways to successful ageing. Mech. Ageing Dev. 130, 553-563.

Deelen, J., Uh, H.W., Monajemi, R., van, H.D., Thijssen, P.E., Bohringer, S., van den Akker, E.B., de Craen, A.J., Rivadeneira, F., Uitterlinden, A.G., Westendorp, R.G., Goeman, J.J., Slagboom, P.E., Houwing-Duistermaat, J.J., Beekman, M., 2011. Gene set analysis of GWAS data for human longevity highlights the relevance of the insulin/IGF-1 signaling and telomere maintenance pathways. Age (Dordr). 2011 Nov 24. doi:10.1007/s11357-011-9340-3.

Di Bona, D., Vasto, S., Capurso, C., Christiansen, L., Deiana, L., Franceschi, C., Hurme, M. Mocchegiani, E., Rea, M., Lio, D., Candore, G., Caruso, C., 2009. Effect of interleukin-6 polymorphisms on human longevity: a systematic review and meta-analysis. Ageing Res. Rev. 8, 36-42.

Flachsbart, F., Caliebe, A., Kleindorp, R., Blanche, H., von Eller-Eberstein, H., Nikolaus, S. Schreiber, S., Nebel, A., 2009a. Association of FOXO3A variation with human longevity confirmed in German centenarians. Proc. Natl. Acad. Sci. U. S. A. 106, 2700-2705

Flachsbart, F., Franke, A., Kleindorp, R., Caliebe, A., Blanche, H., Schreiber, S., Nebel, A. 2010. Investigation of genetic susceptibility factors for human longevity - a targeted nonsynonymous SNP study. Mutat. Res. 694, 9-13.

Flurkey, K., Papaconstantinou, J., Miller, R.A., Harrison, D.E., 2001. Lifespan extension and delayed immune and collagen aging in mutant mice with defects in growth hormone production. Proc. Natl. Acad. Sci. U. S. A. 98, 6736-6741.

Franceschi, C., Motta, L., Valensin, S., Rapisarda, R., Franzone, A., Berardelli, M., Motta, M., Monti, D., Bonafe, M., Ferrucci, L., Deiana, L., Pes, G.M., Carru, C., Desole, M.S. Barbi, C., Sartoni, G., Gemelli, C., Lescai, F., Olivieri, F., Marchegiani, F., Cardelli, M. Cavallone, L., Gueresi, P., Cossarizza, A., Troiano, L., Pini, G., Sansoni, P., Passeri, G., Lisa, R., Spazzafumo, L., Amadio, L., Giunta, S., Stecconi, R., Morresi, R., Viticchi, C., Mattace, R., De, B.G., Baggio, G., 2000. Do men and women follow different trajectories to reach extreme longevity? Italian Multicenter Study on Centenarians (IMUSCE). Aging (Milano) 12, 77-84

Giannakou, M.E., Goss, M., Junger, M.A., Hafen, E., Leevers, S.J., Partridge, L., 2004. Long-lived Drosophila with overexpressed dFOXO in adult fat body. Science 305, 361.

Gueorguiev, M., Lecoeur, C., Meyre, D., Benzinou, M., Mein, C.A., Hinney, A., Vatin, V., Weill, J., Heude, B., Hebebrand, J., Grossman, A.B., Korbonits, M., Froguel, P., 2009. Association studies on ghrelin and ghrelin receptor gene polymorphisms with obesity. Obesity 17, 745-754 (Silver.Spring).

Hampe, J., Wollstein, A., Lu, T., Frevel, H.J., Will, M., Manaster, C., Schreiber, S., 2001. An integrated system for high throughput TaqMan based SNP genotyping. Bioinformatics 17, 654-655.

Harman, D., 1973. Free radical theory of aging. Triangle 12, 153-158.

Herskind, A.M., McGue, M., Holm, N.V., Sorensen, T.I., Harvald, B., Vaupel, J.W., 1996. The heritability of human longevity: a population-based study of 2872 Danish twin pairs born 1870-1900. Hum. Genet. 97, 319-323.

Hjelmborg, J.Vb., Iachine, I., Skytthe, A., Vaupel, J.W., McGue, M., Koskenvuo, M., Kaprio J., Pedersen, N.L., Christensen, K., 2006. Genetic influence on human lifespan and longevity. Hum. Genet. 119, 312-321.

Holzenberger, M., Dupont, J., Ducos, B., Leneuve, P., Geloen, A., Even, P.C., Cervera, P., Le, B.Y., 2003. IGF-1 receptor regulates lifespan and resistance to oxidative stress in mice. Nature 421, 182-187.

Hubacek, J.A., Bohuslavova, R., Skodova, Z., Adamkova, V., 2007. Variants within the ghrelin gene-association with HDL-cholesterol, but not with body mass index Folia Biol. (Praha) 53, 202-206.

Jacobsen, R., Martinussen, T., Christiansen, L., Jeune, B., ndersen-Ranberg, K., Vaupel, J.W., Christensen, K., 2010. Increased effect of the ApoE gene on survival at advanced age in healthy and long-lived Danes: two nationwide cohort studies. Aging Cell 9, 1004-1009.

Kawano, T., Ito, Y., Ishiguro, M., Takuwa, K., Nakajima, T., Kimura, Y., 2000. Molecular cloning and characterization of a new insulin/IGF-like peptide of the nematode Caenorhabditis elegans. Biochem. Biophys. Res. Commun. 273, 431-436.

Kenyon, C.J., 2010. The genetics of ageing. Nature 464, 504-512. 
Kim, D.J., Yi, S.M., Lee, S.Y., Kang, H.S., Choi, Y.H., Song, Y.W., Park, S.C., 2006. Association between the MLH1 gene and longevity. Hum. Genet. 119, 353-354.

Kuningas, M., Mooijaart, S.P., van, H.D., Zwaan, B.J., Slagboom, P.E., Westendorp, R.G., 2008. Genes encoding longevity: from model organisms to humans. Aging Cell 7 , 270-280.

Kuro-o, M., Matsumura, Y., Aizawa, H., Kawaguchi, H., Suga, T., Utsugi, T., Ohyama, Y., Kurabayashi, M., Kaname, T., Kume, E., Iwasaki, H., Iida, A., Shiraki-lida, T., Nishikawa, S., Nagai, R., Nabeshima, Y.I., 1997. Mutation of the mouse klotho gene leads to a syndrome resembling ageing. Nature 390, 45-51.

Kurosu, H., Yamamoto, M., Clark, J.D., Pastor, J.V., Nandi, A., Gurnani, P., McGuinness, O.P., Chikuda, H., Yamaguchi, M., Kawaguchi, H., Shimomura, I., Takayama, Y., Herz, J., Kahn, C.R., Rosenblatt, K.P., Kuro-o, M., 2005. Suppression of aging in mice by the hormone Klotho. Science 309, 1829-1833.

Li, Y., Wang, W.J., Cao, H., Lu, J., Wu, C., Hu, F.Y., Guo, J., Zhao, L., Yang, F., Zhang, Y.X., Li, W., Zheng, G.Y., Cui, H., Chen, X., Zhu, Z., He, H., Dong, B., Mo, X., Zeng, Y., Tian, X.L., 2009. Genetic association of FOXO1A and FOXO3A with longevity trait in Han Chinese populations. Hum. Mol. Genet. 18, 4897-4904.

Lunetta, K.L., D'Agostino Sr., R.B., Karasik, D., Benjamin, E.J., Guo, C.Y., Govindaraju, R Kiel, D.P., Kelly-Hayes, M., Massaro, J.M., Pencina, M.J., Seshadri, S., Murabito, J.M., 2007. Genetic correlates of longevity and selected age-related phenotypes: a genome-wide association study in the Framingham Study. BMC Med. Genet. 8 (Suppl. 1), S13.

Mockett, R.J., Sohal, R.S., Orr, W.C., 1999. Overexpression of glutathione reductase extends survival in transgenic Drosophila melanogaster under hyperoxia but not normoxia. FASEB J. 13, 1733-1742.

Montesanto, A., Latorre, V., Giordano, M., Martino, C., Domma, F., Passarino, G., 2011. The genetic component of human longevity: analysis of the survival advantage of parents and siblings of Italian nonagenarians. Eur. J. Hum. Genet. 19 (8), 882-886.

Nebel, A., Croucher, P.J., Stiegeler, R., Nikolaus, S., Krawczak, M., Schreiber, S., 2005. No association between microsomal triglyceride transfer protein (MTP) haplotype and longevity in humans. Proc. Natl. Acad. Sci. U. S. A. 102, 7906-7909.

Nebel, A., Flachsbart, F., Till, A., Caliebe, A., Blanche, H., Arlt, A., Hasler, R., Jacobs, G. Kleindorp, R., Franke, A., Shen, B., Nikolaus, S., Krawczak, M., Rosenstiel, P., Schreiber, S., 2009. A functional EXO1 promoter variant is associated with prolonged life expectancy in centenarians. Mech. Ageing Dev. 130, 691-699.

Nybo, H., Gaist, D., Jeune, B., Bathum, L., McGue, M., Vaupel, J.W., Christensen, K., 2001 The Danish 1905 cohort: a genetic-epidemiological nationwide survey. J. Aging Health 13, 32-46.

Pan, J., Lin, J., Izzo, J.G., Liu, Y., Xing, J., Huang, M., Ajani, J.A., Wu, X., 2009. Genetic susceptibility to esophageal cancer: the role of the nucleotide excision repair pathway. Carcinogenesis 30, 785-792.

Park, P.U., Defossez, P.A., Guarente, L., 1999. Effects of mutations in DNA repair genes on formation of ribosomal DNA circles and life span in Saccharomyces cerevisiae. Mol. Cell. Biol. 19, 3848-3856.

Pawlikowska, L., Hu, D., Huntsman, S., Sung, A., Chu, C., Chen, J., Joyner, A.H., Schork N.J., Hsueh, W.C., Reiner, A.P., Psaty, B.M., Atzmon, G., Barzilai, N., Cummings, S.R. Browner, W.S., Kwok, P.Y., Ziv, E., 2009a. Association of common genetic variation in the insulin/IGF1 signaling pathway with human longevity. Aging Cell 8, 460-472.

Pawlikowska, L., Hu, D., Huntsman, S., Sung, A., Chu, C., Chen, J., Joyner, A.H., Schork, N.J., Hsueh, W.C., Reiner, A.P., Psaty, B.M., Atzmon, G., Barzilai, N., Cummings, S.R. Browner, W.S., Kwok, P.Y., Ziv, E., 2009b. Association of common genetic variation in the insulin/IGF1 signaling pathway with human longevity. Aging Cell 8, 460-472.
Pedersen, C.B., Gotzsche, H., Moller, J.O., Mortensen, P.B., 2006. The Danish Civil Registration System. A cohort of eight million persons. Dan. Med. Bull. 53, 441-449.

Purcell, S., Neale, B., Todd-Brown, K., Thomas, L., Ferreira, M.A.R., Bender, D., Maller, J., Sklar, P., de Bakker, P.I.W., Daly, M.J., Sham, P.C., 2007. PLINK: a toolset for wholegenome association and population-based linkage analysis. Am. J. Hum. Genet. 81.

Scarpaci, S., Frasca, D., Barattini, P., Guidi, L., Doria, G., 2003. DNA damage recognition and repair capacities in human naive and memory $T$ cells from peripheral blood of young and elderly subjects. Mech. Ageing Dev. 124, 517-524.

Schildkraut, J.M., Iversen, E.S., Wilson, M.A., Clyde, M.A., Moorman, P.G., Palmieri, R.T., Whitaker, R., Bentley, R.C., Marks, J.R., Berchuck, A., 2010. Association between DNA damage response and repair genes and risk of invasive serous ovarian cancer. PLoS One 5, e10061.

Shih, D.M., Gu, L., Xia, Y.R., Navab, M., Li, W.F., Hama, S., Castellani, L.W., Furlong, C.E., Costa, L.G., Fogelman, A.M., Lusis, A.J., 1998. Mice lacking serum paraoxonase are susceptible to organophosphate toxicity and atherosclerosis. Nature 394, 284-287.

Skytthe, A., Kyvik, K., Holm, N.V., Vaupel, J.W., Christensen, K., 2002. The Danish Twin Registry: 127 birth cohorts of twins. Twin Res. 5, 352-357.

Soerensen, M., Dato, S., Christensen, K., McGue, M., Stevnsner, T., Bohr, V.A., Christiansen, L., 2010. Replication of an association of variation in the FOXO3A gene with human longevity using both case-control and longitudinal data. Aging Cell 9, 1010-1017.

Tyner, S.D., Venkatachalam, S., Choi, J., Jones, S., Ghebranious, N., Igelmann, H., Lu, X., Soron, G., Cooper, B., Brayton, C., Hee, P.S., Thompson, T., Karsenty, G., Bradley, A., Donehower, L.A., 2002. p53 mutant mice that display early ageing-associated phenotypes. Nature $415,45-53$.

van der Horst, G.T., van, S.H., Berg, R.J., van Gool, A.J., de, W.J., Weeda, G., Morreau, H., Beems, R.B., van Kreijl, C.F., de Gruijl, F.R., Bootsma, D., Hoeijmakers, J.H., 1997. Defective transcription-coupled repair in Cockayne syndrome B mice is associated with skin cancer predisposition. Cell 89, 425-435.

van Heemst, D., Beekman, M., Mooijaart, S.P., Heijmans, B.T., Brandt, B.W., Zwaan, B.J., Slagboom, P.E., Westendorp, R.G., 2005a. Reduced insulin/IGF-1 signalling and human longevity. Aging Cell 4, 79-85.

van Heemst, D., Mooijaart, S.P., Beekman, M., Schreuder, J., de Craen, A.J., Brandt, B.W., Slagboom, P.E., Westendorp, R.G., 2005b. Variation in the human TP53 gene affects old age survival and cancer mortality. Exp. Gerontol. 40, 11-15.

Van Ness, B., Ramos, C., Haznadar, M., Hoering, A., Haessler, J., Crowley, J., Jacobus, S., Oken, M., Rajkumar, V., Greipp, P., Barlogie, B., Durie, B., Katz, M., Atluri, G., Fang, G., Gupta, R., Steinbach, M., Kumar, V., Mushlin, R., Johnson, D., Morgan, G., 2008. Genomic variation in myeloma: design, content, and initial application of the Bank On A Cure SNP Panel to detect associations with progression-free survival. BMC Med. 6, 26.

Willcox, B.J., Donlon, T.A., He, Q., Chen, R., Grove, J.S., Yano, K., Masaki, K.H., Wilcox, D.C., Rodriguez, B., Curb, J.D., 2008a. FOXO3A genotype is strongly associated with human longevity. Proc. Natl. Acad. Sci. 105, 13987-13992.

Yang, X.R., Pfeiffer, R.M., Wheeler, W., Yeager, M., Chanock, S., Tucker, M.A., Goldstein, A.M., 2009. Identification of modifier genes for cutaneous malignant melanoma in melanoma-prone families with and without CDKN2A mutations. Int. J. Cancer 125, 2912-2917.

Yashin, A.I., De, B.G., Vaupel, J.W., Tan, Q., Andreev, K.F., Iachine, I.A., BonaFe, M., Valensin, S., De, L.M., Carotenuto, L., Franceschi, C., 2000. Genes and longevity: lessons from studies of centenarians. J. Gerontol. A Biol. Sci. Med. Sci. 55, B319-B328.

Yashin, A.I., Wu, D., Arbeev, K.G., Ukraintseva, S.V., 2010. Joint influence of small-effect genetic variants on human longevity. Aging (Albany NY) 2, 612-620. 